WS10-C03

\title{
Accounting for Seismic Energy Release and Fracture Surface Area Development Associated with Hydraulic Fractures
}

\author{
T.I. Urbancic* (Engineering Seismology Group Canada Inc.), A. Cochrane \\ (Engineering Seismology Group Canada Inc.) \& A. Baig (Engineering \\ Seismology Group Canada Inc.)
}

\section{SUMMARY}

Hydraulic fracturing in naturally fractured reservoirs is known to generate seismicity due to the interaction of injected fluids with the pre-existing fracture network. Typically, the observed moment magnitudes for such operations are small, usually with $\mathrm{Mw}<0$. To map the seismicity during these injections, geophones (typically consisting of only $15 \mathrm{~Hz}$ elements) are deployed in arrays in nearby wells. From such configurations information on the relative stimulation volumes and overall fracture dimensions can be obtained. However, the ubiquity of these high-frequency instruments has profound implications for the reliability of magnitude estimates for the largest events associated with these treatments. To address this concern, accelerometers and lower-frequency geophones can be installed to characterize events over a wider magnitude band. Furthermore, these sensors can be combined with the high-frequency downhole geophones to monitor (hybrid sensor network) the full bandwidth of activity that can occur during fracture stimulation programs. 


\section{Amsterdam '14}

\section{Introduction}

Hydraulic fracturing in naturally fractured reservoirs is known to generate seismicity due to the interaction of injected fluids with the pre-existing fracture network. Typically, the observed moment magnitudes for such operations are small, usually with $\mathrm{Mw}<0$. To map the seismicity during these injections, geophones (typically consisting of only $15 \mathrm{~Hz}$ elements) are deployed in arrays in nearby wells. From such configurations information on the relative stimulation volumes and overall fracture dimensions can be obtained. However, the ubiquity of these high-frequency instruments has profound implications for the reliability of magnitude estimates for the largest events associated with these treatments. To address this concern, accelerometers and lower-frequency geophones can be installed to characterize events over a wider magnitude band. Furthermore, these sensors can be combined with the high-frequency downhole geophones to monitor (hybrid sensor network) the full bandwidth of activity that can occur during fracture stimulation programs.

\section{Case Study}

Recently, we have had the opportunity to supplement traditional downhole recording utilizing highfrequency $3 \mathrm{C} 15 \mathrm{~Hz}$ sensors with a sparse eight-station near-surface network consisting of lowfrequency force balance accelerometers $(>0.1 \mathrm{~Hz})$ and $4.5 \mathrm{~Hz}$ geophones associated with hydraulic fracture stimulations in an unconventional shale play in North America. During the stimulation, a total of 4500 events were recorded on the downhole array situated close to the reservoir, ranging in magnitude from M-1 to M-2.6. The near-surface network recorded a total of 28 events ranging from M-0.4 to M1.4; these events were also recorded on the downhole array, however the downhole signals exhibited magnitude saturation affects, which on average, resulted in underestimates of magnitude upwards of 1.8 magnitude units. Significant to the study was the assessment of seismic energy release, which showed that the larger magnitude events detected with the near surface network accounted for a full $71 \%$ of the total seismic energy released during the stimulations. Additionally, these events accounted for a further $11,870 \mathrm{~m} 2$ of activated fracture surface area, approximately $10,295 \mathrm{~m} 2$ more than would have been estimated from the downhole array alone. Overall, the additional surface area as derived from the near-surface network accounts for $<1 \%$ of all the recorded events but adds an additional $4 \%$ to the total liberated surface area when combined with the surface area generated by the 4500 events recorded downhole.

The effectiveness of the stimulation was easily evaluated through this monitoring program. The microseismic events could be used to identify growth from the lower to upper horizon with different pressure rates. The occurrences of larger magnitude events appeared to precede pressure increases in the program, suggesting that larger structures were activated as a result of the injection program even before pressures were increased. This was further exemplified by the inversion results for the $M>0$ events, which were dominated by consistent fracture orientations in alignment with the regional stress field. Interestingly, a small rotation in the principal stresses is apparent resulting from the stimulation program. These observed process sets the foundation to better control and understand stimulation programs.

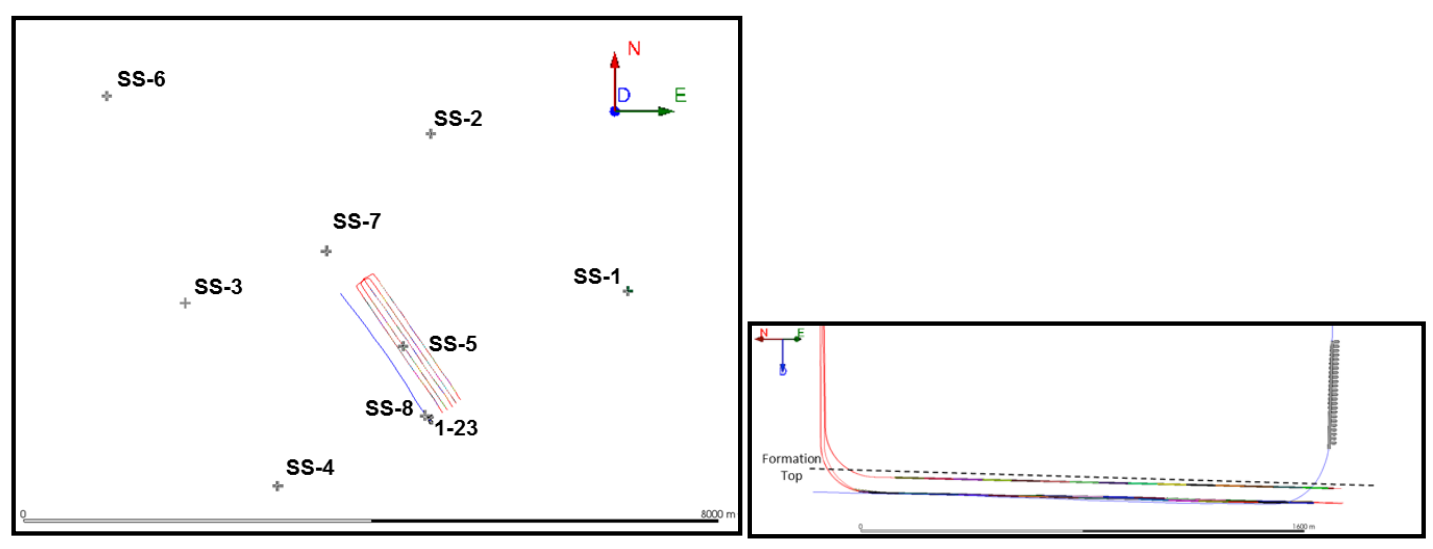




\section{Amsterdam '14}

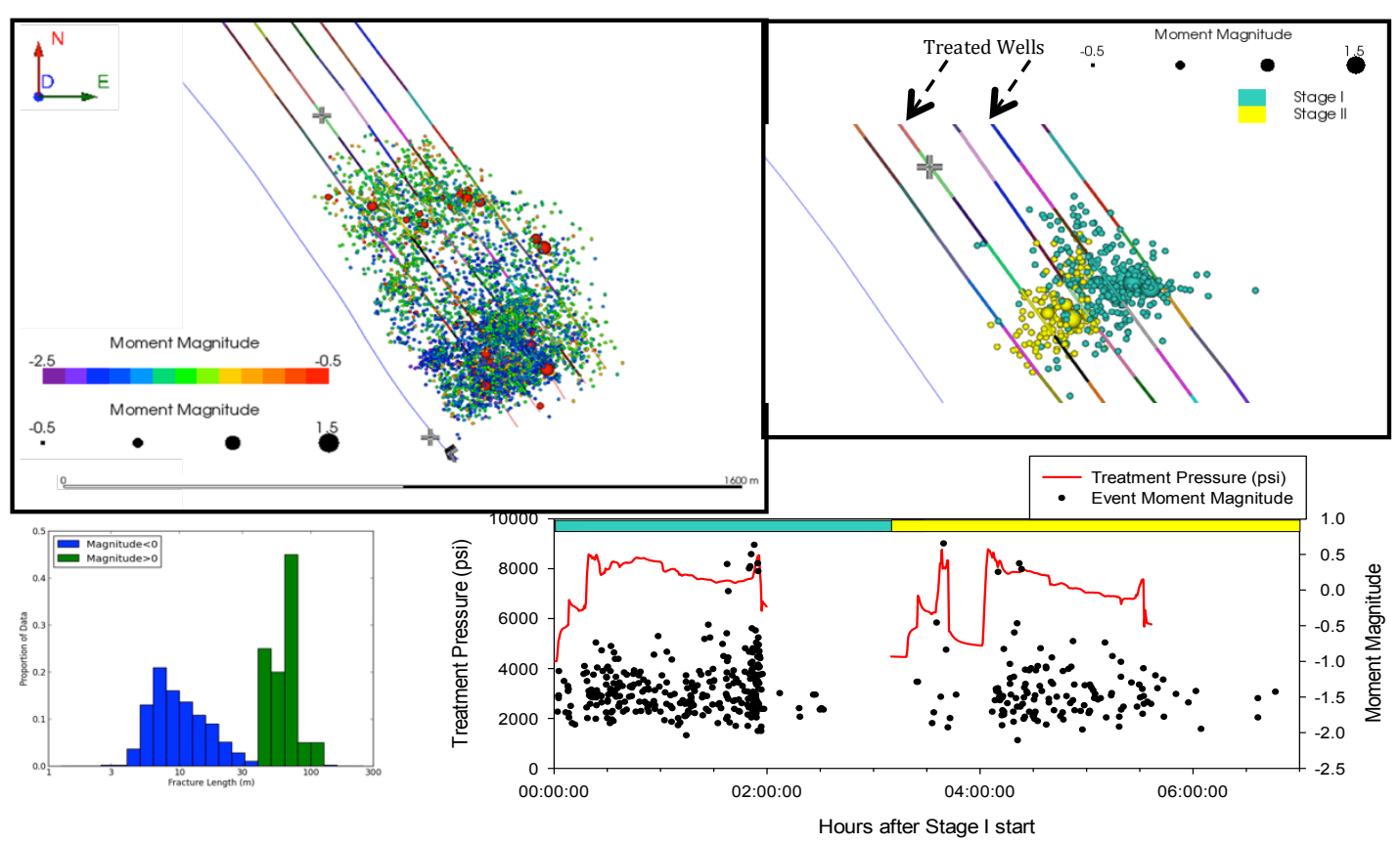

Site location showing near surface network, distribution of seismicity scaled by magnitude, and source radius. Of particular interest is the distribution of events as a function time and magnitude as compared to the injection pressure.

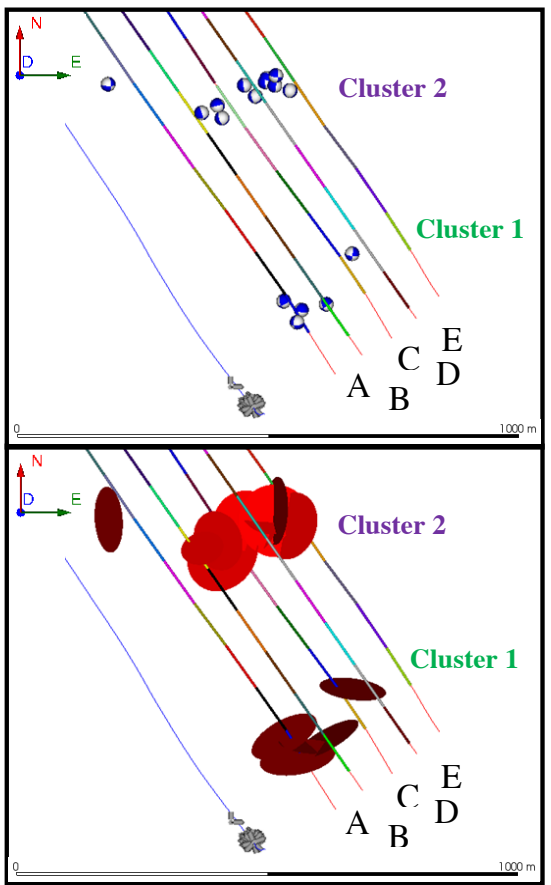

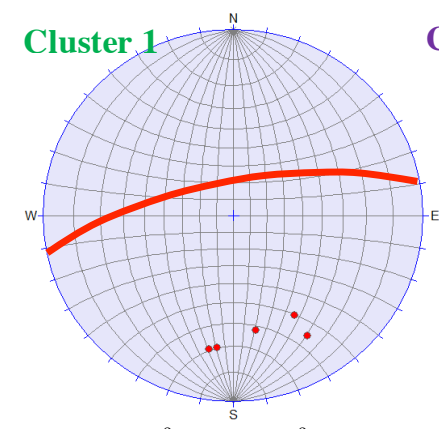

Strike: $259^{\circ}$

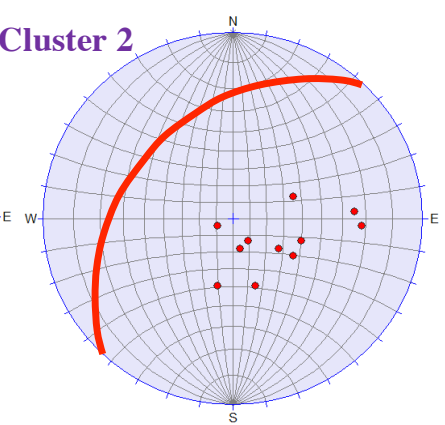

Strike: $223^{\circ}$ Dip: $31^{\circ}$

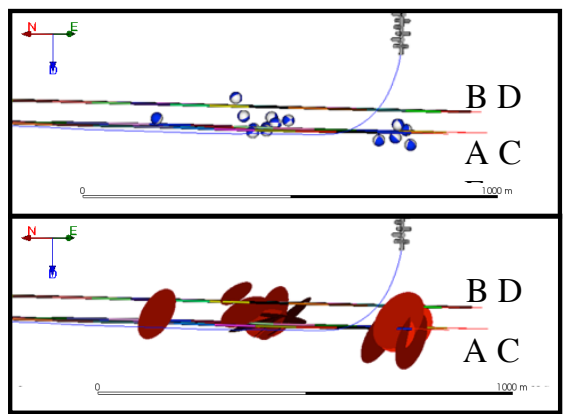

Moment tensor derived solutions for events detected on the near surface network showing the two main fracture sets. The Alignment of the fractures suggests that the principal regional stresses resulted in the activation of faults in cluster 1 , whereas a small rotation due to local stress variations results in the activation of a secondary fault structure in cluster 2. 